(C) Masson, Paris, 1979.

Annales de Parasitologie (Paris)

1979 , t. $54, \mathrm{n}^{\circ}$ 3, pp. 277-295.

\title{
Ecologie des Leishmanioses dans le sud de la France
}

\author{
10. Les formes évolutives de la leishmaniose viscérale canine. \\ Elaboration d'une typologie bio-clinique à finalité épidémiologique.
}

\author{
par G. LANOTTE, J.-A. RIOUX, J. PERIERES et Y. VOLLHARDT \\ (collaboration technique: A. Martini-Dumas) \\ Laboratoire d'Ecologie médicale et Pathologie parasitaire ( $P^{\mathrm{r}}$ J.-A. Rioux), \\ Faculté de Médecine, annexe 163, rue Auguste-Broussonet, F 34000 Montpellier. \\ Laboratoire vétérinaire départemental $\left(D^{r} Y\right.$. Vollhardt), \\ 5, rue Ecole-de-Médecine, F 34000 Montpellier.
}

\section{Résumé.}

Poursuivant l'analyse systémique du foyer leishmanien des Cévennes méridionales, les auteurs exposent le résultat d'une étude des diverses formes, spontanées et provoquées, de la maladie canine ; cette étude basée sur une expérimentation au long cours et sur les données d'enquêtes, prend en compte simultanément, l'évolution clinique, parasitologique et immunologique. Les deux types nosologiques classiques, patent et latent, sont confirmés. Le type latent est lui-même composé de formes précliniques, les plus fréquentes (environ $90 \%$ ), et de formes résolutives (environ $10 \%$ ). L'importance épidémiologique d'une telle distinction est soulignée. Ainsi, les états prépatents, souvent fort longs, peuvent permettre au parasite de se maintenir, plusieurs années durant, sans faire appel à la transmission vectorielle. En fait, l'établissement des fréquences et des durées de ces diverses formes constituent autant d'indices indispensables à l'amélioration des modèles théoriques, actuellement proposés.

\section{Summary.}

Ecology of leishmaniasis in the south of France. 10. Developmental stages and clinical characterization of canine leishmaniasis in relation to epidemiology.

In an analytical study of a focus of leishmaniasis in Southern France (Cévennes), a clini. cal, parasitological and immunological comparison was made of dogs with natural and expe-

Accepté le 30 mars 1979. 
rimental infections of leishmaniasis. Observations were made in longterm laboratory experiments and on dogs found infected in field surveys. Two main clinical forms, patent and latent, were apparent. The latent form could be further subdivided into two types namely preclinical (the commonor: about $90 \%$ ) and resolving (about $10 \%$ ). The epidemiological significance of these distinctions is emphasized. Prepatent states, often lasting for very long periods, can result on the maintenance of the parasites for several years without the need for transmission by the vector. The prevalence and duration of the different forms of disease in dogs are thought to be of great importance in the epidemiology of leishmaniasis in foci in which canids are reservoir hosts.

\section{Introduction}

Chaque jour davantage, l'approche écologique des complexes pathogènes fait appel à l'analyse systémique. Dans cet esprit, les maladies parasitaires, et singulièrement les zoonoses à transmission vectorielle, peuvent être conçues comme des systèmes cycliques composés d'éléments interconnectés (les hôtes), et comme tels, représentés, en première approximation, par des modèles à "compartiments " (J.-M. Legay, 1973). Ainsi en est-il de la leishmaniose viscérale méditerranéenne qui peut être traitée selon un modèle à deux compartiments. Pour ce faire, il suffit de réduire le cycle épidémiologique au mode habituel «Phlébotome $\rightleftarrows$ Chien » et d'établir la liaison " densités vectorielles $\rightleftarrows$ fréquences enzootiques » (J.-A. Rioux et coll., 1974). Sur ces bases, une première tentative de quantification a été entreprise, utilisant les résultats des enquêtes prospectives réalisées en Languedoc-Roussillon (J.-A. Rioux et coll., 1969, H. Croset, 1969 et G. Lanotte et coll., 1974).

Poussant plus loin l'opération, E. Jolivet (1974) a pu proposer un mođ̄èle analytique du type "prédateur-proie ». Cependant, à plusieurs reprises, les difficultés d'une telle entreprise l'ont contraint, pour décrire certaines variations temporelles, à introduire des valeurs hypothétiques, déduites du seul raisonnement logique et non point de l'observation et de l'expérimentation. A cet égard, la prudence manifestée par l'auteur est significative : "Le programme décrit n'est pas rigoureusement exact, le modèle n'étant qu'une première ébauche. Il ne faut considérer que les allures des courbes et non les ordres de grandeur des populations [...]. C'est surtout au niveau de la dynamique de la transmission du parasite qu'il convient de préciser les données, afin de pouvoir décrire la variation des populations de Phlébotomes vecteurs et de Chiens malades [...] par des équations amplement justifiées par la connaissance approfondie des phénomènes. "

Sans prétendre à l'analyse exhaustive du « compartiment Chien », le présent travail répond pro parte au souhait formulé. En fait, il se veut surtout incitatif : son but est de présenter un état descriptif de la sous-population contaminée, en précisant les modalités de passage de l'état infesté à l'état infestant (fig. 1).

Deux approches complémentaires, écologique et expérimentale, ont été utilisées :

L'approche expérimentale, basée sur l'inoculation de chiens sains, a permis de suivre, pas à pas, les diverses formes évolutives: aiguës, subaiguës et chroniques et d'observer le type régressif, objet de nombreuses controverses. 
L'approche écologique, utilisant les enquêtes réalisées en Cévennes, en a confirmé l'existence. L'étude des prévalences séro-immunologiques, calculées par strate de végétation, a montré par ailleurs, la fréquence élevée des formes asymptomatiques, prépatentes ou régressives, à taux d'anticorps infraliminal.

Le mémoire que nous présentons s'ordonne en trois chapitres. Le premier reprend de manière succincte l'histoire de la leishmaniose canine. Le suivant constitue l'essentiel du travail de recherche sous la forme d'une présentation des données, brutes et interprétées. Le dernier propose un essai de synthèse épidémiologique et répond, dans une certaine mesure, à la problématique définie ci-dessus.
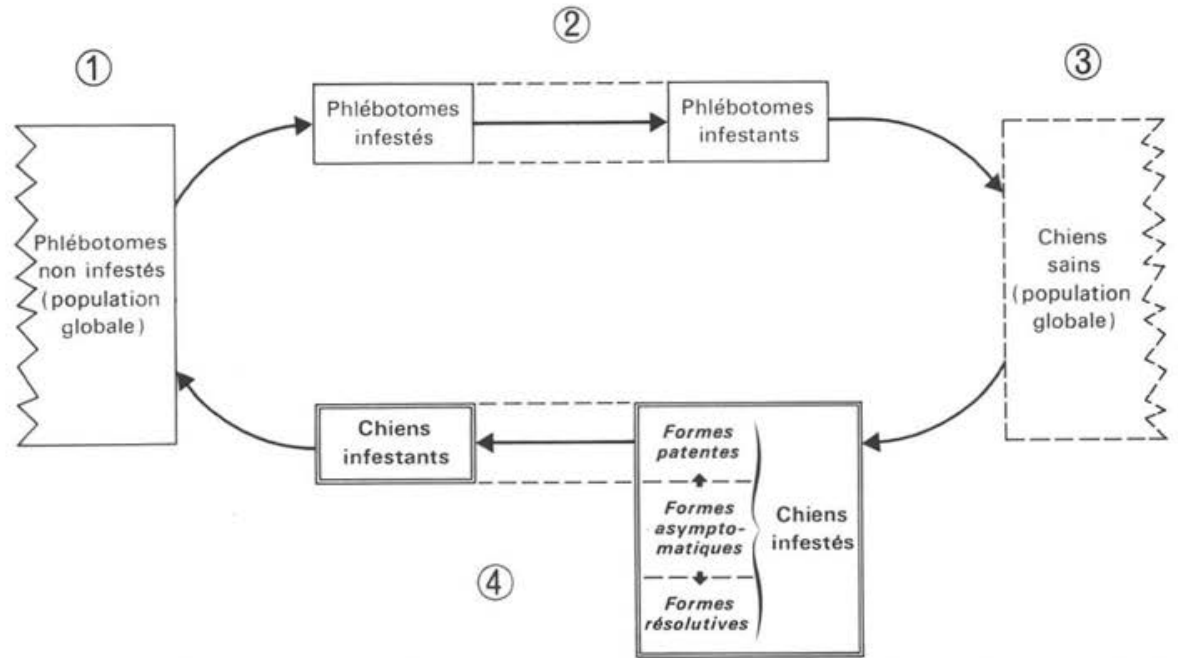

Fig. 1. Schéma du cycle de Leishmania donovani chez le Chien. Seules les formes patentes sont effectivement infestantes pour le vecteur

\section{I. - Rappel historique.}

Depuis la découverte princeps de C. Nicolle (1908), de nombreuses études sur la leishmaniose canine ont été réalisées, précisant la prévalence globale et les fréquences relatives des différentes modalités cliniques.

Les premières descriptions de la maladie canine sont dues à C. Basile (1910) qui distingue, en Italie, les types aigüs et chroniques, types retrouvés en Tunisie par A.-C. Gray (1913) et en Algérie, par G. Lemaire et coll. (1913).

En 1933, P. Giraud et H. Cabassu en France font le point des diverses expressions cliniques. Ils authentifient les deux formes précédentes, ajoutent la forme latente et confirment les possibilités de guérison signalées par A. Laveran et A. Petit (1909) et par E. Pringault (1917). Dès lors, nombreux sont les auteurs, français ou étrangers, 
qui reprennent ces travaux et insistent, à leur tour, sur la fréquence des formes asymptomatiques. Ainsi, sur 37 chiens leishmaniens dépistés par E. Falchetti et G. Faure-Brac (1932) dans la région de Nice, la moitié ne présente aucun signe clinique. A Malte, S. Adler et O. Theodor $(1932,1935)$ font des constatations identiques : sur 1500 chiens infectés, $60 \%$ paraissent en bonne santé. En Espagne, C. Sanchez-Botija (1936) conclue dans le même sens. En Grèce, B. Malamos (1947) trouve $50 \%$ des chiens parasités d'apparence normale. Les formes asymptomatiques sont encore signalées en Ouzbékistan (N.-J. Khodukin, 1943) et au Brésil (L.-M. Deane, 1955).

En France, plusieurs mises au point ont été consacrées à ce problème (J. Ranque et coll., 1948, 1975, 1978). Récemment. R. Sanchis et coll. (1976) ont retrouvé, grâce à l'inoculation expérimentale, les diverses expressions spontanées: sur quatre chiens infestés, deux ont présenté une leishmaniose viscérale grave, les deux autres, une forme latente.

\section{II. - Évolution immuno-clinique de la leishmaniose canine.}

\section{1. - Leishmaniose canine expérimentale.}

L’étude a porté sur quatre chiens d'élevage, inoculés avec de fortes doses de formes amastigotes et régulièrement suivis aux plans clinique, immunologique et parasitologique, pendant des périodes variant de trois mois à sept ans.

\section{A. MATÉRIEL ET MÉTHODES.}

L'inoculation est conduite, selon la méthode de L.-A. Stauber (1955) modifiée, à l'aide de formes amastigotes d'origine canine : le tissu parasité est dissocié au mortier de Potter. Un frottis, réalisé avec le broyat, est coloré (May-Grunwald-Giemsa); dans le même champ de microscope sont dénombrés à la fois les noyaux cellulaires et les leishmanies. Le rapport leishmanies/noyaux est établi sur un minimum de 500 noyaux. Parallèlement, le nombre de noyaux cellulaires par unité de volume est calculé par comptage à la cellule de Thoma.

A l'occasion de chaque prise de sang, un examen clinique est pratiqué, permettant de noter l'évolution des signes cutanés, fonctionnels et généraux.

La surveillance parasitologique (1) est assurée par :

— une hémoculture bi-hebdomadaire ou hebdomadaire,

- un examen du suc ganglionnaire, effectué dès l'apparition d'une adénopathie, repris trois mois plus tard, puis tous les six mois,

- une étude histo-pathologique (examen sur coupe et sur empreintes, cultures NNN) des divers organes, lors de l'autopsie.

Le suivi séro-immunologique est basé sur la technique d'immunofluorescence indirecte quantitative. Le taux initial des anticorps est déterminé lors de l'inoculation, puis surveillé régulièrement, d'abord de manière rapprochée (quatre à sept jours), puis tous les quinze jours.

(1) L'analyse proprement dite comprend l'examen direct sur frottis coloré (May-Grunwald-Giemsa) et la mise en culture sur milieu NNN (contrôle au $6^{e}$ jour, suivi de cinq repiquages successifs en cas de négativité). 


\section{B. RÉSultats}

Les modalités évolutives, tant cliniques que biologiques, permettent de classer chaque animal dans les quatre rubriques suivantes: forme aiguë, forme subaiguë viscérale, forme chronique viscéro-cutanée, forme régressive.

\section{- Forme aiguë évolutive.}

Elle est exprimée chez le chien $C$ : corniaud, mâle âgé de 19 mois, inoculé le 16-12-71 par la voie intradermique en 10 points, au niveau de la truffe, des oreilles et de la queue (souche C-3732. Dose totale : 110 millions de parasites). Le sang est prélevé tous les quatre jours. La recherche des leishmanies est effectuée dans le suc ganglionnaire ( $32^{\circ}$ et $48^{e}$ jour) et dans les lésions cutanées $\left(20^{\circ}\right.$ et $48^{\circ}$ jour).

Dès le $20^{\circ}$ jour, l'animal présente une lésion érythémato-papuleuse aux points d'inoculation. Les « boutons" augmentent rapidement de volume $(\varnothing=2 \mathrm{~cm})$ puis s'ulcèrent $\left(45^{\circ}\right.$ jour $)$. Parallèlement, se développe une dépilation diffuse accompagnée d'une hypertrophie unguéale. Une adénopathie modérée est constatée dès la fin du premier mois. L'animal meurt au $127^{\circ}$ jour dans un état cachectique après un coma de quelques jours. Les lésions cutanées demeurent stériles jusqu'à la phase ulcérative ; elles deviennent très riches en leishmanies à partir du $48^{c}$ jour. Cependant, les parasites sont présents dans le ganglion pré-scapulaire dès le $32^{e}$ jour. A l'autopsie, ils sont uniquement retrouvés dans la rate. Les hémocultures restent constamment négatives. Au plan immunologique, les anticorps fluorescents sont dépistés dès le $8^{c}$ jour $(1 / 10)$. Leur ascension est lente mais régulière (fig 2$)$. Le maximum est atteint à la $12^{\circ}$ semaine $(1 / 20480)$. Le taux se maintient autour du $1 / 10240$ jusqu'à la dernière semaine, pour chuter brutalement $(1 / 2560$ puis $1 / 640)$ quelques heures avant l'exitus.

En définitive, la maladie s'est exprimée sur le mode aigu, emportant l'animal en quatre mois. Les parasites ont été mis en évidence aux points d'inoculation, dans les ganglions et dans la rate. Les anticorps sont apparus précocément, pour atteindre un niveau élevé et persister jusqu’à la mort.

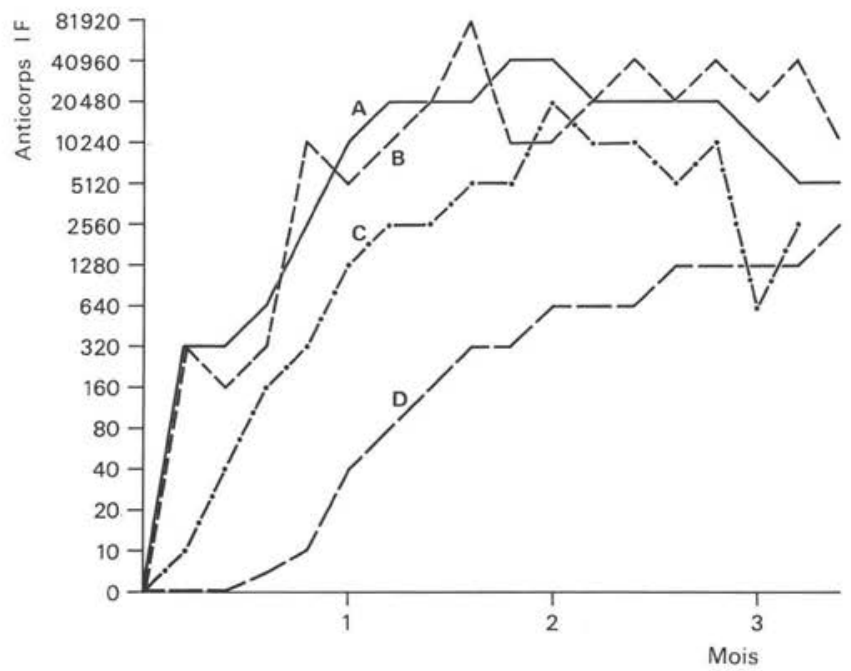

Fig. 2. Leishmaniose canine expérimentale. Evolution des anticorps fluorescents jusqu'au troisième mois. Aucune relation n'est mise en évidence entre l'inclinaison de la pente et la gravité de la maladie. Dans le groupe des courbes supérieures voisinent, en effet, une forme résolutive (chien $\mathrm{A}$ ) et une forme consomptive (chien $\mathrm{C}$ ) 


\section{- Forme subaiguë à dominante viscérale.}

Elle est illustrée par le chien B: corniaud, femelle âgée de 19 mois, inoculée le 16-12-71, par les voies sous-cutanées, intra-péritonéales, intra-veineuses et intra-hépatiques (souche C3732. Doses respectives: $83,83,55$ et 55 millions de parasites). La surveillance sérologique s'effectue tous les quatre jours pendant 20 semaines, tous les huit jours pendant les $140 \mathrm{se}$ maines suivantes, puis tous les 15 jours. Quatre ponctions ganglionnaires sont réalisées au $6^{\circ}, 9^{\circ}, 12^{\circ}$ et $15^{\circ}$ mois. Un prélèvement cutané est effectué au $12^{\circ}$ mois.

Au plan clinique, l'état général reste satisfaisant jusqu'au $8^{e}$ mois. A cette époque, on constate une discrète anémie cependant qu'apparaissent une hypertrophie unguéale et une dépilation diffuse. Progressivement, se développe un amaigrissement, accompagné d'une polyadénopathie et d'ulcérations multiples.

Le premier examen parasitologique, pratiqué au $6^{\circ}$ mois (ganglion poplité), demeure négatif. Le parasite est cependant détecté aux $9^{\circ}$ et $15^{\circ}$ mois (ganglion) ainsi qu'au $12^{\circ}$ mois (peau). Lors de l'autopsie, les leishmanies sont mises en évidence dans la rate, le foie, le cœur, les ovaires et les ganglions. Le sang demeure stérile.

Les anticorps, présents dès le $4^{c}$ jour $(1 / 10)$, évoluent en trois phases (fig. 3$)$ : une phase d'ascension (fig. 2) jusqu'au taux maximal de 1/81 920 ( $9^{\circ}$ semaine), une phase en plateau, d'une durée de 33 semaines, prolongée par une phase de décroissance, jusqu'à la mort survenue à la $68^{c}$ semaine $(1 / 2560)$.

En résumé, ce chien a présenté une forme subaiguë classique, à prédominance viscérale. La phase prépatente a duré huit mois environ. Au cours de l'évolution, les leishmanies ont été détectées dans les ganglions et les lésions cutanées. A l'autopsie, elles ont été retrouvées dans tous les viscères. Les anticorps fluorescents ont rapidement atteint un taux important $\left(65^{e}\right.$ jour $)$, puis régressé légèrement, pour se maintenir à un niveau élevé jusqu'à l'exitus.

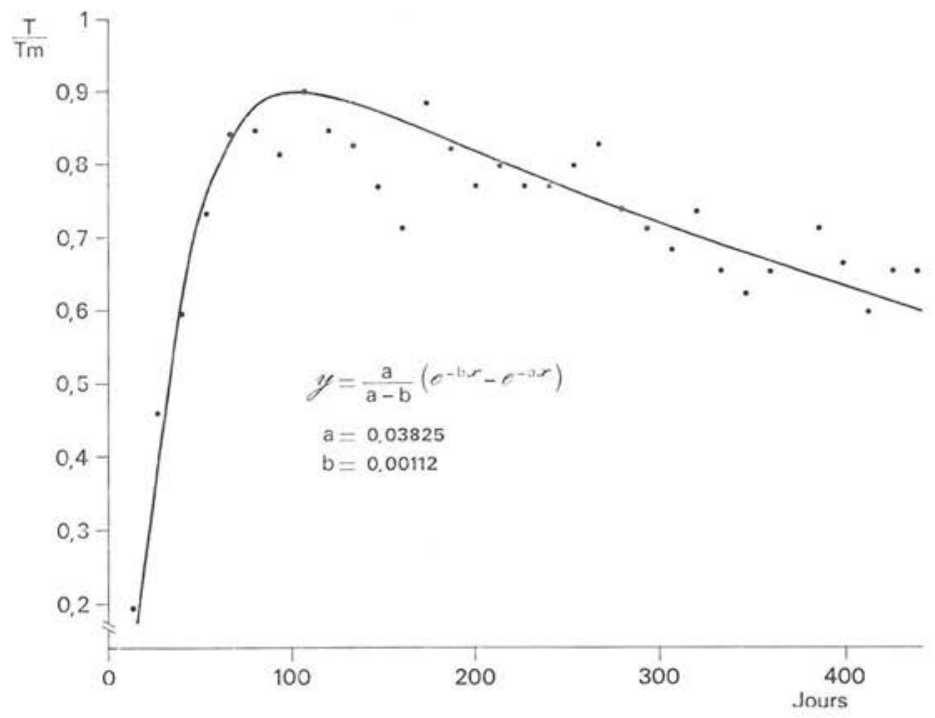

Fig. 3. Dynamique des anticorps fluorescents dans la leishmaniose canine expérimentale (chien B, forme subaiguë viscéro-cutanée). Dans la première phase de la maladie, la distribution des taux peut être exprimée par une différence exponentielle (modèle catalytique de Cohen) 


\section{- Forme chronique viscéro-cutanée.}

Cette forme est observée chez le chien $D$ : corniaud, mâle âgé de 17 mois, inoculé le 27-4-74, par voie intrapéritonéale (souche C-3728 d'origine canine, entretenue sur Atlantoxerus getulus. Dose : 28 millions de parasites). Les examens sérologiques sont réalisés tous les huit jours pendant la première année, puis tous les quinze jours. La présence de parasites dans le ganglion est contrôlée à six reprises $\left(5^{\circ}, 17^{\circ}, 21^{\circ}, 30^{\circ}, 44^{\circ}\right.$ et $50^{\circ}$ mois).

Pendant les quatre premières années, aucun signe clinique ne se manifeste, à l'exception d'une discrète adénopathie (septième mois), persistant inchangée depuis sa constatation.

Vers le cinquantième mois, apparaissent une parésie du train postérieur, des ulcérations des coudes, une kératite et de volumineuses adénopathies préscapulaires. Les symptômes restent stationnaires jusqu'à ce jour $\left(57^{\circ}\right.$ mois).

Au plan parasitologique, les leishmanies ne sont retrouvées que dans les ganglions.

Le anticorps fluorescents sont dépistés au $21^{\circ}$ jour $(1 / 5)$. Leur ascension s'effectue lentement jusqu'au taux maximal de $1 / 10240$, atteint à la $18^{e}$ semaine (fig. 2). Dès lors les titres s'abaissent pour se maintenir autour de $1 / 1280$.

Il s'agit donc d'une forme chronique, dans laquelle les signes viscéraux (parésie du train arrière) oculaire (kératite) et cutanés (ulcérations) apparaissent tardivement (quatrième année). Les leishmanies sont cependant détectées dans le ganglion bien avant l'expression clinique. La courbe d'évolution des anticorps est du même type que celle du chien B, avec toutefois une moindre ascension des taux.

\section{- Forme latente régressive.}

Cette forme est matérialisée par le chien $A$ : corniaud, mâle âgé de 19 mois, inoculé le 16-12-71, par les voies sous-cutanée, intra-péritonéale, intra-veineuse et intra-hépatique (souche C-3732. Doses respectives: $165,165,110$ et 110 millions de parasites). La surveillance sérologique est calquée sur celle du chien $\mathrm{B}$. Les contrôles parasitologiques sont effectués à six reprises $\left(6^{\circ}, 9^{\circ}, 15^{\circ}, 21^{\circ}, 29^{\circ}\right.$ et $40^{\circ}$ mois $)$.

Au cours des six premières années, l'animal ne présente d'autre signe qu'une adénopathie poplitée transitoire (du $6^{e}$ au $34^{e}$ mois). En septembre $1977\left(69^{e}\right.$ mois) apparaît une dépilation discrète du museau et des oreilles, accompagnée d'un léger amaigrissement. Aucune adénopathie n'est alors notée. En un mois ces signes s'estompent jusqu'à la restitutio ad integrum qui se maintient actuellement.

Les leishmanies ne sont observées, dans le suc ganglionnaire, qu'au sixième et neuvième mois. Les quatre prélèvements suivants restent négatifs. Les hémocultures sont constamment stériles.

Les anticorps fluorescents sont décelés dès le $4^{e}$ jour $(1 / 40)$. Ils évoluent en trois temps : progression (fig. 2) jusqu'à la $7^{\circ}$ semaine (1/40 960), oscillations autour du $1 / 20480$ pendant 10 semaines, baisse par palliers. A l'issue de la $7^{\circ}$ année, leur niveau atteint le 1/80 (fig. 4).

Ce chien a donc présenté une forme régressive, émaillée d'un épisode clinique spontanément résolutif. La maladie ne s'est d'ailleurs révélée que par la présence épisodique de parasites dans les ganglions périphériques et par l'existence d'anticorps fluorescents durant les sept années d'observation.

Au triple point de vue, clinique, parasitologique et immunologique, les observations précédentes peuvent être résumées comme suit :

Au plan clinique, quatre formes évolutives se sont exprimées :

- une forme aiguë (chien C), consomptive ; 


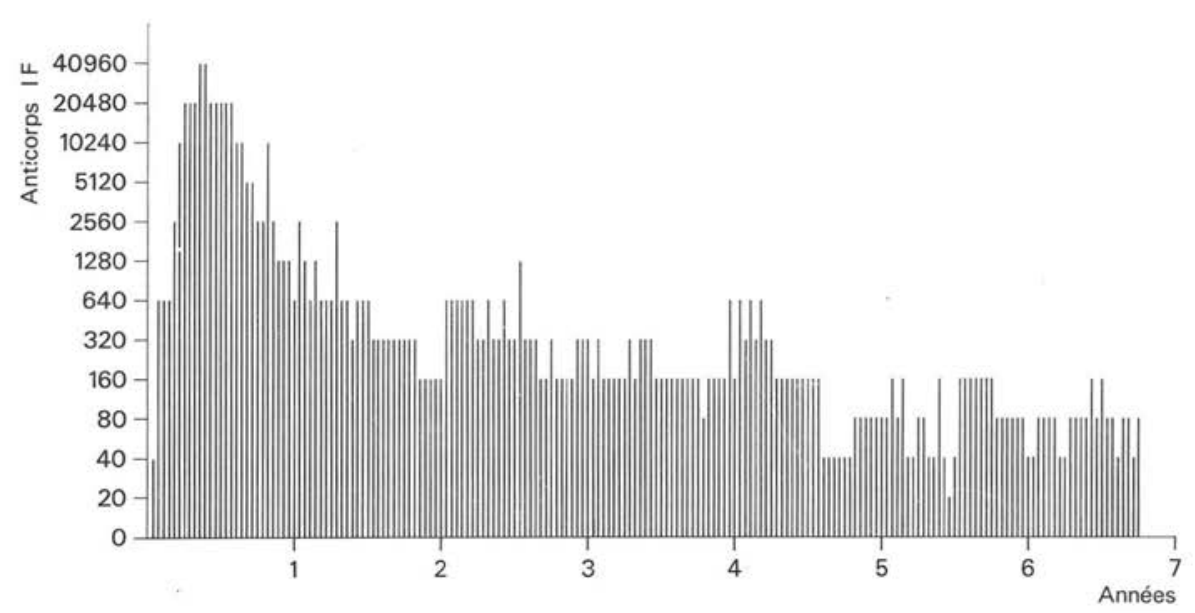

Fig. 4. Leishmaniose canine expérimentale. Dynamique des anticorps fluorescents dans la forme régressive (chien $\mathrm{A}$ ). Après une montée rapide (taux maximal: 1/40 960), la courbe s'infléchit pour se stabiliser, vers la cinquième année, aux environs de 1/80

- une forme subaiguë (chien B), associant un syndrome général (anémie, amaigrissement, polyadénopathie) à des signes cutanés caractéristiques (dépilation, onychogryphose, ulcérations) ;

- une forme chronique (chien D), à longue période de latence. Dans ce cas, outre les lésions cutanéo-muqueuses (ulcérations) et oculaires (kératite), une parésie du train arrière, au demeurant classique, s'est manifestée en période terminale ;

- une forme latente et spontanément résolutive (chien A), émaillée d'un épisode clinique mineur. Alors que le type précédent se rapproche de la forme subaiguë (persistance des parasites dans les ganglions, maintien d'un taux élevé d'anticorps), cette forme paraît nettement individualisée (régression puis disparition des adénopathies, baisse significative des taux d'anticorps).

Au plan parasitologique, la présence de leishmanies dans les ganglions périphériques constitue un trait commun aux quatre formes : le parasite est détecté dès l'apparition des adénopathies ou peu de temps après.

Au plan immunologique, un fait s'impose : l'élaboration rapide des anticorps fluorescents (entre le quatrième et le vingt-et-unième jour) et leur production tout au long de l'évolution. Ce stigmate constitue un indice fidèle, susceptible de témoigner de l'infection au stade prépatent et de persister après la disparition des signes cliniques. Au surplus, la dynamique générale des anticorps permet de distinguer quatre phases évolutives (fig. 2, 3 et 4): une période de début, immunologiquement muette, une phase ascensionnelle, de type exponentiel, plus courte chez les animaux massivement infestés, une phase en plateau, de durée variable, s'ordonnant autour d'un niveau maxi- 
mal, enfin une phase de régression, volontiers oscillante. La durée de la première phase peut être exprimée en jours, celle de la deuxième en semaines ; les suivantes se comptent en mois, voire en années.

\section{2. - Leishmaniose canine spontanée.}

Les chiens leishmaniens pris en compte dans cette étude proviennent soit d'enquêtes systématiques, soit de clientèle vétérinaire. Au total, nous avons disposé d'une série assez importante de cas instantanés (152 chiens) dont certains ont pu être suivis pendant tout ou partie de leur évolution (42 chiens).

\section{A. MATÉriel et mÉthodes.}

Les enquêtes systématiques ont été menées en Cévennes méridionales, dans une zone de $4000 \mathrm{~km}^{2}$, en forme de rectangle à grand axe perpendiculaire à la côte. Les examens ont été effectués sur 5423 chiens. Toutefois, sur ce nombre, 1712 animaux vivaient réellement dans les biotopes riches en Phlébotomes cynophiles. Tous les sujets porteurs d'anticorps fluorescents ont été soumis à un ou plusieurs contrôles parasitologiques, immunologiques et cliniques.

Les chiens de clientèle sont, le plus souvent, adressés au Laboratoire par les praticiens de la région Languedoc-Roussillon. Ils font partie des nombreux cas dépistés annuellement par notre secteur Immunologie : les vétérinaires souhaitent, avant tout, une confirmation parasitologique du diagnostic sérologique. Quelques uns, parmi les animaux dépistés, nous sont cependant confiés pour être traités et surveillés dans notre Service.

\section{B. RÉsultats.}

La leishmaniose canine " naturelle » se distribue en deux groupes de formes, patentes et latentes. Dans le premier, nous incluons les formes traitées. Le second réunit, en fait, une majorité d'états prépatents ; deux cas seulement constituent la fraction spontanément résolutive.

\section{- La leishmaniose canine patente. Aspects immuno-cliniques.}

\section{- Leishmaniose non traitée.}

Habituellement, les animaux sont adressés en consultation (fig. 5) pour une dépilation $(85 \%)$ prédominante à la tête (museau, oreille, tour des orbites) et aux lombes. Souvent ils présentent une croissance exagérée des ongles $(73 \%)$, gênant l'animal à la marche. A ces signes d'appel s'associe, de manière constante, une adénopathie localisée (poplitée) ou multiple (jugulaire). Dans la moitié des observations, sont notées des ulcérations de petite taille, parfois difficiles à déceler (plis interdigitaux). Plus rarement, ces troubles s'accompagnent de kératite (20\%), d'hémorragies (7\%), de manifestations nerveuses $(7 \%)$ ou de vomissements $(6 \%)$. La présence simultanée de symptômes spécifiques est fréquente (fig. 6) ; leur association avec des signes généraux (amaigrissement, asthénie, fièvre) est hautement évocatrice. 


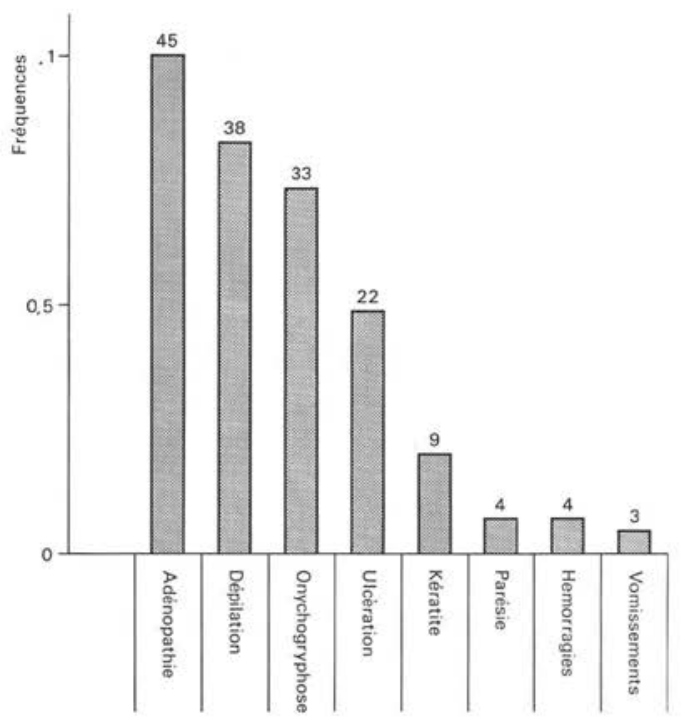

Fig. 5. Leishmaniose canine spontanée. Fréquence des principaux symptômes

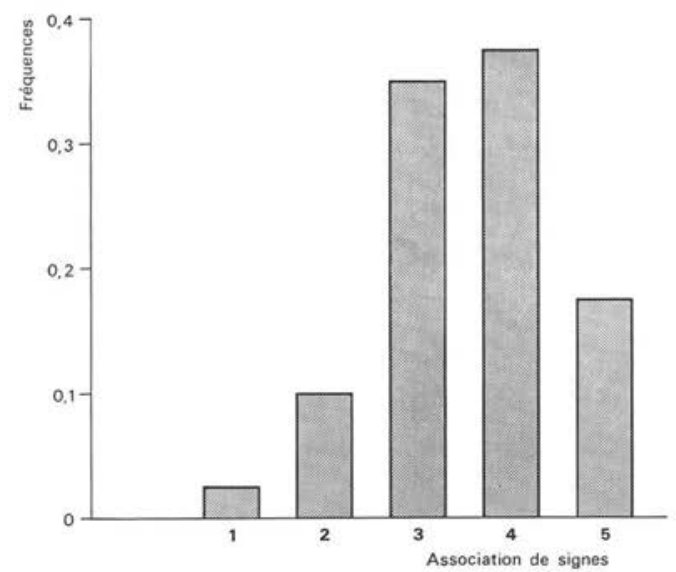

Fig. 6. Leishmaniose canine spontanée. Fréquence des associations de symptômes

Les taux d'anticorps fluorescents atteignent généralement des valeurs élevées (fig. 7). Dans près de $70 \%$ des cas, ils se situent au niveau ou au-dessus du $1 / 1280$. La moyenne géométrique de l'inverse des titres (GRMT) est de même ordre (1237). Toutefois, les différentes valeurs calculées semblent indépendantes des symptômes cliniques ou de leur association. 


\section{- Leishmaniose traitée.}

La thérapeutique utilise un composé antimonié (Glucantime ${ }^{\circledR}$ ) ou un dérivé des diamidines (Lomidine ${ }^{\circledR}$ ), administré en cure de vingt jours, à raison de deux ou trois cures par an. Un traitement d'entretien d'une injection mensuelle de Lomidine ${ }^{\circledR}$ peut être instauré entre les cures proprement dites.

Dans le cas général, les signes cliniques s'estompent dès la première cure et disparaissent après la deuxième. Une période de rémission, d'une durée de quelques mois à un an, peut en imposer pour la guérison. Toutefois, la récidive est quasi constante. Les épisodes cliniques deviennent alors subintrants : l'animal meurt à l'issue de la dernière rechute, dans un état cachectique, emporté par une complication infectieuse, hémorragique ou rénale. Dans quelques cas (10\% environ), le traitement n'a aucune incidence sur les symptômes cliniques : la maladie évolue alors sur le mode aigu. La durée de vie probable (1) d'un chien, dépisté et traité au stade viscéro-cutané, est de 22 mois. Ainsi, sur 20 chiens régulièrement suivis, trois n'ont pas survécu plus de six mois, deux n'ont pas dépassé un an, six sont morts au cours de la deuxième année, six au cours de la troisième, un au cours de la quatrième et deux au cours de la cinquième.

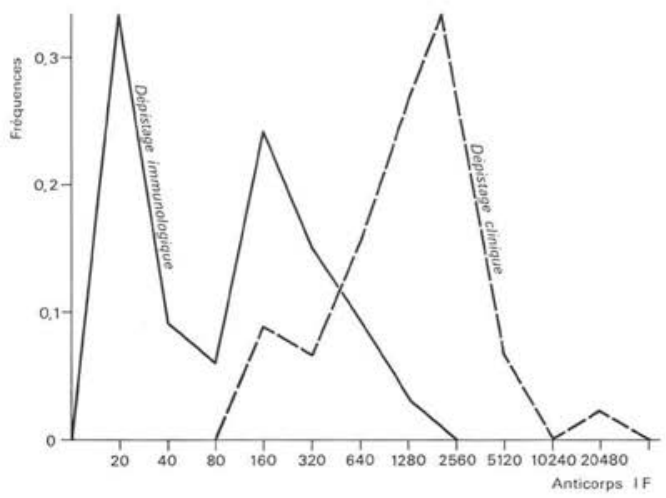

Fig. 7. Les deux types de distributions, uni et bimodal, des anticorps fluorescents dans la leishmaniose canine spontanée

En trait continu: Cas dépistés au cours d'enquêtes prospectives. La distribution bimodale témoigne de l'existence de deux sous-populations. Le premier mode $(1 / 20)$ correspond aux formes asymptomatiques (pré-patentes et régressives), le deuxième (1/160) aux formes évolutives.

En trait discontinu: Cas dépistés au cours des consultations vétérinaires. Ici, la distribution est du type unimodal. Le décalage du mode vers les forts taux d'anticorps $(1 / 2560)$, traduit l'existence d'une majorité de formes évolutives.

Comme on peut s'y attendre (S. Adler et O. Theodor, 1932), le traitement n'a que peu d'action sur la vitalité du parasite : dans dix autopsies complètes pratiquées,

(1) La durée de vie probable ou vie médiane (R. Dajoz, 1974) est l'âge au-delà duquei $50 \%$ de la population concernée a disparu. 
tous les viscères renfermaient des leishmanies vivantes (cultures sur NNN). Trois de ces chiens ont d'ailleurs infesté massivement le vecteur habituel de la leishmaniose en Cévennes: Phlebotomus ariasi, Tonnoir, 1921 (J.-A. Rioux et coll., 1972).

Au long des cures, les taux d'anticorps varient parallèlement à l'intensité de la maladie. Ils s'abaissent notablement pendant les phases de rémission pour remonter lors des rechutes. Les fluctuations des GRMT rendent compte de cette évolution (tableau I).

Tableau I. Leishmaniose canine traitée par Glucantime ${ }^{\circledR}$. On constate une remontée des anticorps fluorescents (GRMT) après la troisième cure.

\begin{tabular}{|c|c|c|c|c|c|}
\hline $\begin{array}{c}\text { Nombre } \\
\text { de chiens }\end{array}$ & $\begin{array}{c}\text { Temps moyen } \\
\text { (en mois) } \\
\text { entre } \\
\text { les cures }\end{array}$ & $\begin{array}{c}\text { Dépistage } \\
\text { (GRMT) }\end{array}$ & $\begin{array}{c}1^{\text {re }} \text { cure } \\
\text { (GRMT) }\end{array}$ & $\begin{array}{c}2^{\mathrm{e}} \text { cure } \\
\text { (GRMT) }\end{array}$ & $\begin{array}{c}3^{\mathrm{e}} \text { cure } \\
\text { (GRMT) }\end{array}$ \\
\hline 9 & $\begin{array}{c}\text { (GRMT) } \\
4\end{array}$ & 1382 & 806 & 345 & 640 \\
\hline
\end{tabular}

Ainsi, la leishmaniose canine traitée peut être considérée comme une forme clinique autonome. Son évolution est émaillée par une succession de rechutes, caractérisées au plan immunologique par une variation sinusoïdale des taux d'anticorps. La phase terminale rappelle cependant la leishmaniose non traitée, tant au plan symptomatique que parasitologique.

\section{- La leishmaniose canine latente.}

Les enquêtes menées dans le foyer cévenol, à l'aide de la réaction d'immunorescence, ont permis de dépister 33 leishmanioses latentes sur un total de 67 cas (49\%). Dans ce groupe, le niveau atteint par les anticorps est généralement bas (GRMT : 91) ; le tiers des sujets ne dépasse pas le $1 / 20$ au moment du premier examen (fig. 7). L'évolution, suivie sur 22 chiens, s'est terminée par une leishmaniose-maladie, à l'exception de deux cas qui sont demeurés infra-cliniques. Nous commentons ces diverses éventualités :

\section{- Leishmaniose latente, stade pré-clinique d'une forme viscéro-cutanée évolutive.}

Les 20 animaux concernés par cette forme ont atteint le stade clinique dans les mois (deux à neuf) suivant le dépistage. Au cours de la phase asymptomatique les taux d'anticorps se sont sensiblement modifiés, sans toutefois atteindre des valeurs très élevées. Extraits de ce lot, trois chiens ont pu être régulièrement suivis au Laboratoire ; en voici les observations :

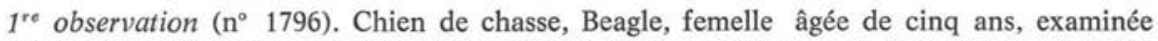
le 23-1.71 à l'occasion d'un dépistage systématique. Présence d'anticorps fluorescents au 1/80. Absence de signes cliniques. Le 16-6-71, soit cinq mois plus tard, constatation d'une adénopathie poplitée, de laquelle est isolée le parasite. Les anticorps ont atteint le 1/160. Au cours 
du sixième mois d'observation, apparaissent des signes cutanés (dépilation, onychogryphose, ulcération des coudes); le taux d'anticorps se maintient au $1 / 160$. Onze mois après le dépistage, soit cinq mois après le début clinique, les anticorps sont parvenus au taux de $1 / 1280$; la symptomatologie demeure inchangée. A partir du $22^{e}$ mois, s'installe un amaigrissement progressif, accompagné d'une micropolyadénopathie. Les anticorps persistent au même taux. La mort survient le 26-11-73, à savoir 33 mois après le premier examen. Les leishmanies sont alors présentes dans tous les viscères; le taux d'anticorps est de 1/5 120 .

$2^{\circ}$ observation $\left(\mathrm{n}^{\circ}\right.$ 14700). Chien de chasse, Fauve de Bretagne, mâle âgé de 6 ans. Lorsqu'il est examiné pour la première fois (6-9-77), le taux d'anticorps est élevé (1/640). Aucun signe clinique n'est noté à l'exception d'une légère adénopathie qui permet de confirmer le diagnostic par l'examen direct et la culture. Au cinquième mois, l'état clinique et immunologique est stationnaire. Deux mois plus tard, le taux d'anticorps augmente légèrement. Le premier symptôme clinique apparaît vers le huitième mois, sous la forme d'une dépilation furfuracée localisée à la tête. Dès lors, l'évolution se précipite; en un mois la dépilation s'est généralisée, au point de donner à l'animal l'aspect dit de "chien nu ». La mort survient le 7-11-78, soit treize mois après le dépistage immunologique et cinq mois après le début des signes cutanés. Les anticorps ont atteint le 1/2 560. A l'autopsie, les leishmanies sont présentes dans tous les viscères.

$3^{e}$ observation ( $\mathrm{n}^{\circ}$ 14692). Chien de compagnie, corniaud, mâle, âgé de 13 ans, dépisté le 6-9-77. Absence de symptômes cliniques. Taux d'anticorps : 1/160. Quatre mois plus tard, ce taux atteint 1/1280. La maladie se développe alors sur le mode viscéro-cutané habituel. La mort survient le 25-5-78, soit huit mois après le dépistage. La rate, le foie, le cœur, le rein et les surrénales contiennent des leishmanies vivantes. Le taux d'anticorps demeure inchangé: $1 / 1280$.

Ainsi, dans les trois cas présentés, les symptômes cliniques se sont manifestés quatre à huit mois après le dépistage séro-immunologique, lors de la phase ascensionnelle des anticorps. Ceux-ci se sont maintenus à un niveau élevé jusqu'à l'exitus, survenu dans un délai variant de cinq à seize mois. A l'autopsie, les parasites abondaient dans la plupart des viscères.

- Leishmaniose latente, spontanément résolutive.

Cette forme, soupçonnée mais rarement observée, a pu être identifiée chez deux chiens dépistés à l'occasion d'enquêtes catamnestiques, réalisées de 1972 à 1976 sur le versant méridional des Cévennes. Les parasites ont été mis en évidence une seule fois, dans les ganglions périphériques. Placés sous observation continue pendant trois et sept ans, les animaux ont présenté quelques épisodes cliniques, toujours brefs et de faible intensité. Les anticorps fluorescents n'ont jamais complètement disparu, mais les taux, oscillants, sont toujours restés faibles. Voici le résumé de ces observations :

$4^{\circ}$ observation $\left(\mathrm{n}^{\circ}\right.$ 1377). Chien de berger, corniaud, femelle âgée de neuf ans. A l'examen, le 1-12-70, il présente un bon état général et n'accuse aucun signe cutané. Le test séroimmunologique révèle un taux d'anticorps fluorescents au 1/160. La recherche des leishmanies dans le suc ganglionnaire est négative. Le 2-6-71, l'examen clinique met en évidence une importante adénopathie poplitée, ainsi qu'un intertrigo de la patte antérieure droite. Le taux d'anticorps atteint le 1/640. La ponction ganglionnaire est positive. Jusqu'en octobre 1971, l'état clinique reste stationnaire mais le taux d'anticorps redescend progressivement pour parvenir au 1/160 en juillet 1971. Le 4-10-71 apparaissent de nouveaux signes cliniques (ulcération des coudes, kératite, iritis), signes qui régressent progressivement alors que le taux d'anti- 
corps continue d'osciller entre le $1 / 160$ et le 1/640. Les examens parasitologiques de trois ponctions ganglionnaires (janvier, mars, mai 1973) et d'un prélèvement cutané demeurent négatifs. En mars 1973, on note l'apparition d'une tumeur mammaire; l'animal s'affaiblit peu à peu tandis que la tumeur se développe. Le 27-10-74, la mort survient. Le chien est alors âgé de 13 ans. Le taux d'anticorps est de 1/160. A l'autopsie, la recherche systématique des leishmanies par l'examen direct et la culture des différents viscères demeure négative.

$5^{c}$ observation $\left(\mathrm{n}^{\circ}\right.$ 1708). Chien truffier, corniaud, mâle âgé de deux ans, dépisté le 9-2-71 dans la même région que l'animal précédent, présente des adénopathies poplitées discrètes. Taux d'anticorps fluorescents: $1 / 320$. Revu quatre mois plus tard (9-6-71), il ne manifeste toujours pas de signe clinique, viscéral ou cutané. La recherche des leishmanies est négative. Le taux d'anticorps n'est plus que de 1/80. Le 8-11-71, une nouvelle ponction ganglionnaire révèle la présence de leishmanies (culture). Un pityriasis ainsi qu'une dépilation diffuse sont alors notés. Les anticorps sont au 1/80. Cependant, l'animal reste vif et prend du poids, alors que les signes cliniques disparaissent, à l'exception de l'adénopathie. En décembre 1972, le taux d'anticorps est remonté au $1 / 320$, toujours sans modification de l'état général et cutané. Dans le courant de l'année 1973, l'adénopathie poplitée régresse à son tour. Le taux d'anticorps fluctue entre le $1 / 40$ et le $1 / 80$. Trois examens du suc ganglionnaire sont négatifs. Depuis cette date, l'état clinique, parasitologique et immunologique reste inchangé. Actuellement l'animal, âgé de neuf ans, est toujours en excellent état. Sa maladie ne s'exprime plus que par une trace immunologique $(1 / 40)$.

Ainsi, la typologie des formes cliniques, établie à partir de l'étude expérimentale, peut être appliquée à la maladie spontanée. Toutefois, dans les conditions naturelles, la montée initiale des anticorps fluorescents paraît plus lente : parmi les chiens dépistés en période pré-clinique, onze étaient porteurs d'anticorps au $1 / 20$, taux caractérisant les premiers jours de la leishmaniose expérimentale. Or, nos enquêtes ont toujours été réalisées de novembre à mars, c'est-à-dire bien au-delà de la période d'activité des vecteurs. Ces constatations amènent à admettre que, dans la nature, la période d'incubation est relativement longue ou, tout au moins, que le début de la maladie clinique est nettement plus insidieux que ne le laisseraient supposer les données de l'expérimentation. Par ailleurs, chez certains sujets, les signes cliniques apparaissent précocément, au cours de la montée des anticorps. L'explication de ces phénomè-

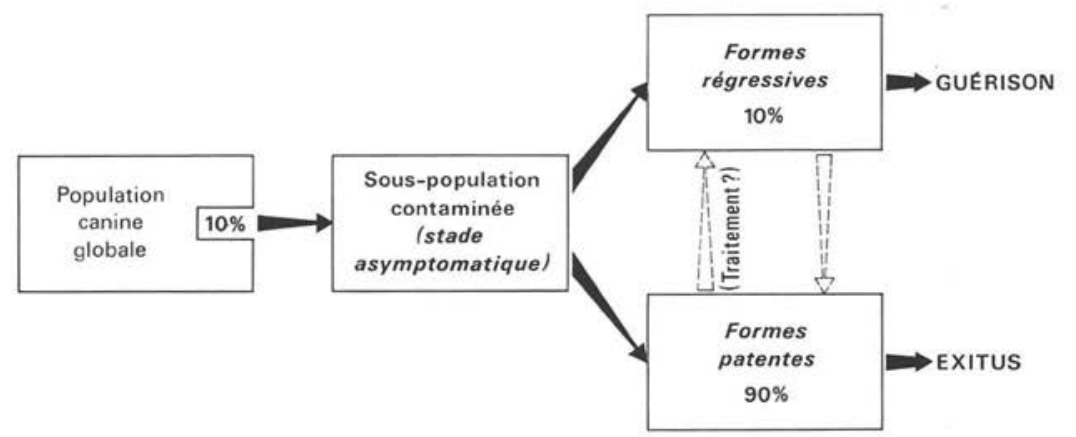

Fig. 8. Indices de fréquences et successions des diverses formes de leishmaniose canine spontanée. Les indices ont été établis à partir des données recueillies dans la strate «la plus active ” (chênaie mixte) du foyer cévenol 
nes est certainement à rechercher dans les différences de virulence et de porte d'entrée de l'agent infectieux. Ainsi, dans la leishmaniose naturelle, la quantité de parasites introduits par le vecteur doit être très faible. D'autre part, la voie intradermique oppose, vraisemblablement, une barrière plus efficace à la diffusion du germe que les autres voies parentérales. Enfin, les formes promastigotes, issues du cycle intra-vectoriel (stades métacycliques), sont, sans doute, au plan de la pathogénicité, différentes des formes amastigotes.

En définitive, sur le terrain, s'observent deux types de formes: patentes et infracliniques. Les premières se caractérisent par un taux d'anticorps élevé et l'existence de symptômes viscéro-cutanés. Les secondes, à taux d'anticorps faibles, regroupent deux types évolutifs, l'un se transformant en quelques mois en forme viscéro-cutanée, l'autre régressant progressivement jusqu'à la guérison.

\section{III. - Commentaires et conclusion.}

Certes, le schéma proposé ne saurait être considéré comıme définitif. Il réclamerait, en particulier, d'être étayé par une analyse immuno-pathologique plus approfondie. Tel qu'il est néanmoins, il peut être largement utilisé, tant au plan clinique qu'épidémiologique.

Ainsi, dans la leishmaniose naturelle comme dans la leishmaniose expérimentale, le suivi des anticorps fluorescents apporte une information précieuse sur le pronostic des formes latentes. Il permet de surveiller pas à pas l'évolution générale de la maladie, alors que les stigmates cliniques et parasitologiques n'existent pas encore ou ont disparu.

Cependant, le principal intérêt de ce travail est d'ordre épidémiologique : la prise en compte du schéma expérimental rend, en effet, possible l'identification et l'interprétation, en termes de "risque», des diverses formes cliniques observées in natura. Pour ce qui concerne le foyer étudié on peut, d'ores et déjà, disposer d'un ordre de grandeur des prévalences instantanées, relatives aux trois groupes de sujets contaminés (1). Ainsi les cas «infestants " (phase patente) représenteraient $50 \%$ de l'ensemble de la sous-population atteinte, les cas "pré- ou pauci-infestants " (phase prépatente), $40 \%$ et les cas «non infestants " (phase régressive) $10 \%$ (fig. 8).

De plus, grâce aux données séro-immunologiques, il est possible d'évaluer de manière plus correcte le pourcentage de sujets contaminés au sein de la population globale. Quels que soient les titres observés, le nombre de porteurs d'anticorps s'élève, en effet, lorsque l'on progresse des zones périphériques vers la zone centrale du foyer (fig. 9). On peut, de la sorte, calculer la fréquence des cas correspondant à un contact parasitaire effectif : dans la strate la plus active du foyer (chênaie mixte), elle est de

(1) En raison du petit nombre de cas dépistés, il ne s'agit pas d'exprimer ces prévalences en termes de probabilité mais de les considérer seulement comme des indices, susceptibles d'aider à une meilleure compréhension du cycle tant au plan structural que fonctionnel. 
l'ordre de $10 \%$ et représente, par conséquent, le double du chiffre retenu jusqu’à présent (tableau II).

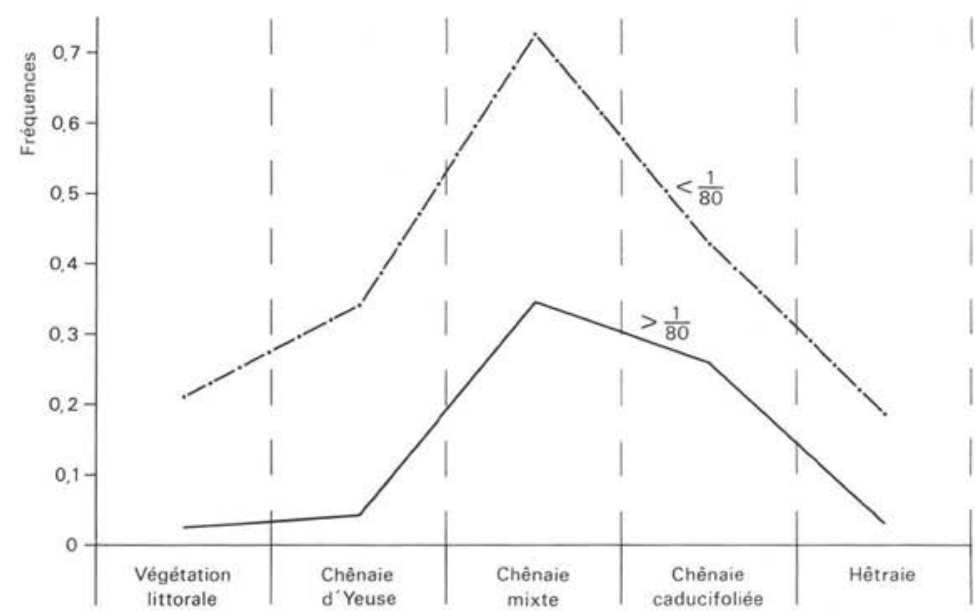

Fig. 9. Leishmaniose canine spontanée. Fréquence des anticorps fluorescents selon les étages de végétation (foyer cévenol). Le parallélisme des deux distributions $(<1 / 80$ et $>1 / 80)$ dénote l'existence d'un nombre non négligeable (environ $50 \%$ ) de leishmanioses authentiques, mais "laissées pour compte" (fréquences: en \%).

En revanche, il est beaucoup plus difficile d'apprécier « le temps de passage » de la forme pré-clinique à la forme patente. Et cependant, ce délai est important, car il détermine le potentiel de la dissémination géographique du parasite. Seule, peut être estimée une "durée minimale moyenne ", laquelle est d'environ de 10 mois (2).

Tableau 2. Leishmaniose canine: récapitulatif des indices épidémiologiques calculés à l'occasion de la présente étude.

\begin{tabular}{|c|c|}
\hline $\begin{array}{l}\text { Fréquence de la leishmaniose canine dans la zone d'activité } \\
\text { maximale du foyer } \ldots \ldots \ldots \ldots \ldots \ldots \ldots \ldots \ldots \ldots \ldots \ldots \ldots \ldots \ldots \ldots \ldots \ldots \ldots \ldots\end{array}$ & 0,1 \\
\hline Fréquences relatives des formes évolutives : & \\
\hline - forme patente $\ldots \ldots \ldots \ldots \ldots \ldots \ldots$ & 0,5 \\
\hline - forme prépatente $\ldots \ldots \ldots \ldots \ldots \ldots$ & 0,4 \\
\hline - forme régressive $\ldots \ldots \ldots \ldots \ldots \ldots \ldots$ & 0,1 \\
\hline Incubation minimale moyenne $\ldots \ldots \ldots \ldots$ & 10 mois \\
\hline «Vie médiane» d'un chien traité . & 22 mois \\
\hline
\end{tabular}

(2) En considérant que les chiens se sont contaminés au cours du mois d'août précédant la date d'apparition des premiers signes immunologiques. 
A l'image de nombreux cycles parasitaires, celui de la leishmaniose viscérale méditerranéenne ne peut être abordé correctement sans une analyse approfondie du comportement parasitaire dans les divers hôtes, vecteurs et vertébrés. L'objet du présent travail est précisément d'apporter une contrib-ation à la connaissance intrinsèque du réservoir canin. Poursuivie pendant huit ans, à la fois au laboratoire (expérimentation) et sur le terrain (enquêtes), l'étude entreprise a permis de caractériser les diverses formes évolutives, considérées sous leur triple aspect, clinique, parasitologique et immunologique. Malgré les difficultés techniques, les résultats obtenus ont permis d'élaborer une typologie réaliste, distinguant quatre formes, deux patentes (traitée et non traitée) et deux latentes (préclinique et résolutive). Une telle typologie doit pouvoir être utilisée avec fruit dans les modèles épidémiologiques actuellement proposés.

\section{REMERCIEMENTS}

Aux nombreux vétérinaires praticiens de la région Languedoc-Roussillon qui n'ont jamais cessé de nous manifester leur bienveillant intérêt, à notre collègue R. Killick-Kendrick qui nous a toujours conseillé avec pertinence, nous adressons nos plus vifs remerciements.

\section{Bibliographie}

Adler S., Theodor O. (1932) : Investigations on Mediterranean Kala-azar. VI. Canine viscéral leishmaniasis. Proc. R. Soc., 110, 402-412.

Adler S., Theodor O. (1935) : Investigations on Mediterranean Kala-azar. VII. Further observations on canine visceral leishmaniasis. Proc. R. Soc., 116, 494-504.

Basile C. (1910) : Alcune osservazioni sulla presenza di leishmania nei cani (nota preliminate). Atti della Reale Academia del Lincei. R.C. Ist. sup. Sanita, Roma, 19, 158-160.

Cardamatis J. (1912) : Leishmaniose du Chien en Grèce. Bull. Soc. Pathol. Exot., 5, 88-89.

Carrieu M., Rambault A., Venel H., Harant H. (1934) : La Leishmaniose canine dans la région de Montpellier. Arch. Soc. Sci. Med. Biol. Montpellier, 194-197.

Croset H. (1969) : Ecologie et systématique des Phlebotomini (Diptera, Psychodidae) dans deux foyers, français et tunisien, de leishmaniose viscérale. Essai d'interprétation épidémiologique. Thèse Sciences, Montpellier, $516 \mathrm{p}$.

Curasson G. (1943) : Traité de Protozoologie vétérinaire et comparće. T. II. Vigot, édit., Paris, 330 p.

Dajoz R. (1974). Dynamique des populations. Collection Ecologie, Masson édit., Paris, 297 p.

Deane L. M. (1956) : Leishmaniose visceral no Brasil; estudos sobre reservatorios e transmissores realizados no Estado do Cearà. Thesis Medicina Sao-Paulo. Serv. nacional educaçào sanitaria, Edit., Rio-de-Janeiro, 162 p.

Deane L. M., Deane M.P. (1955) : Observaçoes preliminares sobre a importancia comparativa do homen, do cao e da raposa (Lycalopex vetulus) como reservatorios da Leishmania donovani em area endemica do calazar, no Cearà. O Hospital, 48, 61-76.

Dedet J.-P., Ben Osman F., Chadli A., Croset H., Rioux J.-A. (1973): La leishmaniose canine en Tunisie. Fréquences actuelles de l'enzootie d'après une enquête séro-immunologique. Ann. Parasitol. Hum. Comp., 48, 653-660.

Donatien A., Lestoquard F. (1935): Observations et réflexions sur la leishmaniose générale du chien. Arch. Inst. Pasteur, Alger, 13, 320-338.

Donatien A., Lestoquard F. (1938) : Sur l'évolution de la leishmaniose viscérale du chien. Arch. Inst. Pasteur Alger, 16, 191-202.

Falchetti E., Faure-Brac G. (1932): La leishmaniose canine à Nice. Etude épidémiologique. Bull. Soc. Pathol. Exot., 25, 1091-1099. 
Giauffret A., Sanchis R., Vitu C. (1976) : Les examens de laboratoire dans la leishmaniose canine. I. Application au diagnostic, au pronostic et au contrôle du traitement dans la maladie naturelle. Revue Med. Vet., 127, 913-930.

Giauffret A., Taliercio Y.P. (1971): Mise en évidence d'anticorps sériques chez le chien atteint de leishmaniose par une technique d'immunofluorescence indirecte. Recl. Med. Vet. Ec. Alfort, 147, 141-151.

Giraud P., Cabassu H. (1933): Le chien est-il le réservoir de virus de la leishmaniose interne? Arch. Med. Gen. Colon., 2, 23-27.

Giraud P., Raybaud J. (1932) : Kala-azar grave chez un enfant de deux ans, leishmaniose latente chez un chien en contact avec l'enfant. Bull. Soc. Pediat., Paris, 30, 574-579.

Gray A.-C. (1913): Leishmaniose naturelle du Chien à Tunis. Arch. Inst. Pasteur Tunis, 6, 102105.

Groulade P. (1965): Clinique canine. T. I, Maloine, édit., Paris, 394 p.

Jolivet E. (1974): Essai de modélisation analytique du développement d'un foyer de leishmaniose canine. In: Ecologie des leishmanioses. Colloque Internationaux du C.N.R.S., $\mathrm{n}^{\circ}$ 239, Montpellier, 18-24 août 1974 , 307-310.

Kerbœuf D. (1973) : Diagnostic immunologique de la leishmaniose canine. Thèse Vét., Alfort, 161 p.

Khodukin N. J. (1943) : Focal distribution of visceral leishmaniasis in Tashkent for twenty years and the problem of the reservoir of infection. Med. Parazit. Moscow, 12, 61-69.

Lanotte G., Rioux J.-A., Croset H., Vollhardt Y. (1974): Ecologie des leishmanioses dans le Sud de la France. 7. Dépistage de l'enzootie canine par les méthodes immunosérologiques. Ann. Parasiiol. Hum. Comp., 49, 41-62.

Lanotte G., Rioux J.-A., Croset H., Vollhardt Y. (1974) : Dépistage de la leishmaniose canine. Stratégie d'enquête utilisée dans le foyer des Cévennes méridionales. In: Ecologie des leishmanioses. Colloques Internationaux du C.N.R.S., $\mathrm{n}^{\circ}$ 239, Montpellier, 18-24 août 1974, 117-128.

Lanotte G., Rioux J.-A., Croset H., Vollhardt Y. (1978) : Ecologie des leishmanioses dans le Sud de la France. 9. Les méthodes d'échantillonnage dans le dépistage et l'analyse de l'enzootie canine. Ann. Parasitol. Hum. Comp., 53, 33-45.

Laveran A. (1917): Leishmanioses. Kala-azar, bouton d'Orient, leishmaniose américaine. Masson, édit., Paris, 515 p.

Laveran A., Petit A. (1909): Infections expérimentales légères ou latentes du singe et du chien par le Kala-azar tunisien. Bull. Soc. Pathol. Exot., 2, 584-587.

Legay J.-M. (1973) : La méthode des modèles. Etat actuel de la méthode expérimentale, 1-73. Introduction à l'étude des modèles à compartiments, 117-145. Informatique et Biosphère, édit., Lille, Paris, $145 \mathrm{p}$.

Lemaire G., Sergent E., L'Héritier A. (1913): Recherches sur la leishmaniose du Chien d'Alger. Bull. Soc. Pathol. Exot., 6, 579-581.

Lépine P., Bilfinger F. (1936): Recherche de la leishmaniose viscérale chez le Chien de fourrière d'Athènes. Bull. Soc. Pathol. Exot., 29, 131-135.

Malamos B. (1947): Leishmaniasis in Greece. Trop, Dis. Bull., 44, 1-7.

Nicolle C. (1908): Reproduction expérimentale du Kala-azar chez le Chien. Origine canine probable de cette affection. Bull. Soc. Pathol. Exot., 1, 188-190.

Nicolle C., Comte C. (1908) : Origine canine du Kala-azar. Arch. Inst. Pasteur Tunis, 2, 59-62.

Nicolle C., Comte C. (1909): Kala-azar expérimental du Chien. Arch. Inst. Pasteur Tunis, 1, 38-42.

Nicolle C., Comte C. (1909) : Kala-azar expérimental du Chien. Arch. Inst. Pasteur Tunis, 3, $136-138$.

Nicolle C., Comte C. (1909) : Kala-azar expérimental du Chien. Arch. Inst. Pasteur Tunis, 4, 199-200.

Nicolle C., Comte C. (1910): Kala-azar expérimental du Chien. Quelques observations nouvelles et quelques faits nouveaux. Immunité par première atteinte après guérison complète. Arch. Inst. Pasteur Tunis, 3, 103-107.

Pringault E. (1914): La leishmaniose canine à Marseille. Bull. Soc. Pathol. Exot., 1, 484.488.

Pringault E. (1917): Recherches sur la leishmaniose canine. Thèse Médecine, Montpellier, 57 p.

Quilici M., Dunan S., Ranque J. (1968) : L'immunofluorescence dans les leishmanioses. Comparaison avec la réaction de fixation du complément. Med. Trop., 28, 37-43. 
Ranque J., Ranque M., Cabassu J., Cabassu H. (1948): Le diagnostic précoce de la leishmaniose canine par la ponction ganglionnaire. Réflexions à propos de soixante examens positifs obtenus en dix mois dans la région marseillaise. Bull. Acad. Nat. Med., 132, 339-340.

Ranque J., Quilici M., Bellendy P., Dunan S. (1978) : Les réservoirs de virus de la leishmaniose viscérale en Provence. Med. Trop., 58, 405-409.

Ranque J., Quilici M., Dunan S. (1975): Les leishmanioses du Sud-est de la France. Ecologie, Epidémiologie, Prophylaxie. Acta Tropica, 32, 371-80.

Rioux J.-A., Croset H. et Lanotte G. (1974): Ecologie d'un foyer méditerranéen de leishmaniose viscérale. Esasi de modélisation. In: Ecologie des leishmanioses. Colloques Internationaux du C.N.R.S., $\mathrm{n}^{\circ}$ 239, Montpellier, 18-24 août 1974, 295-305.

Rioux J.-A., Golvan Y.-J., Croset H., Tour S., Houin R., Abonnenc E., Petitdidier M., Vollhardt Y., Dedet J.-P., Albaret J.-L., Lanotte G., Quilici M. (1969): Epidémiologie des leishmanioses dans le sud de la France. Monographie I.N.S.E.R.M., n $\mathrm{n}^{\circ}$ 37, 223 p.

Rioux J.-A., Lanotte G., Croset H., Dedet J.-P. (1972): Ecologie des leishmanioses dans le Sud de la France. 5. Pouvoir infestant comparé des diverses formes de leishmaniose canine vis-à-vis de Phlebotomus ariasi Tonnoir, 1921. Ann. Parasitol. Hum. Comp., 47, 413-419.

Sanchez Botija C. (1936) : Leishmaniosis canina. Algunas observaciones sobre los caracteres generales del foco endemico en Madrid. Trab. Inst. Biol. Animal., 2, 70-87.

Sanchis R., Vitu C., Giauffret A. (1976) : Les examens de laboratoire dans la leishmaniose canine. II. Evolution des tests biologiques dans la maladie expérimentale. Revue Med. Vet., 127, 1191-1202.

Sergent Ed., Sergent Et. (1910) : Kala-azar. Existence de la leishmaniose chez les chiens d'Alger. Bull. Soc. Pathol. Exot., 3, 510-511.

Stauber L.A. (1955): Leishmaniasis in the hamster. In: Some physiological aspects and consequences of parasitism. W. H. Cole, édit., New Brunswick, pp. 76-90.

Yakimoff W. L., Kohl-Yakimoff N. (1911): La leishmaniose canine. Arch. Inst. Pasteur Tunis, 4, 249-257. 